\title{
Parallelism of Source Text with Target Text in News Translation
}

\author{
Ninuk Sholikhah Akhiroh \\ \{ninuk.akhiroh@mail.unnes.ac.id\} \\ Department of Sociology and Anthropology, Universitas Negeri Semarang ${ }^{1}$
}

\begin{abstract}
News translation is questionable as part of translation by a number of translation scholars. This paper aims to give solutions to this problem. Using some leading opinions on the nature of news translation confronted with a preliminary research done by the author, this paper proposes the following solutions. Parallelism of source text with target text should be the first to be confirmed. Source text must be able to be traced and compared to as one to one translation. This means that the translated text has a function, text structure and meaning that can be compared in parallel with the source text. The next thing to be confirmed is the use of holistic approach in doing news translation research. The complexity of intra and extralinguistic factors in news translation should be covered by using a proper combination of linguistic approach with other related non-linguistic approach.
\end{abstract}

Keywords: News translation, Parallelism, Source text, Target text

\section{Introduction}

News translation is a popular practice in today's global communication, where people's need for information from various parts of the world is increasing. Information flows from one point to various other points through global news which is localized in the target language. This confirms the role of news translation in international news distribution. Therefore, news translation research is worth studying.

Compared to other types of translation, for example the translation of literary works, news translation is still categorized as an infant. Valdeon [1] states that academic interest in news translation research began in the 1980s and 1990s, and its publication showed a significant increase since the mid-2000s. The clearer position of journalistic translation studies in translation research is also indicated by the inclusion of a special chapter on news translation in several references to translation studies, such as "The Handbook of Translation Studies" (Benjamin, 2010), and "The Routledge Handbook of Translation Studies" (Millan \& Bartrina, 2013).

The rise of news translation studies conducted by experts raises a number of problems and criticisms that arise from the uniqueness of news translation in particular and journalistic translation in general. Davier \& Van Doorslaer [2] call this phenomenon a challenge in journalistic translation research by referring to the opinions of a number of researchers, such as Bielsa and Bassnett (2019), Davier (2014, 2017), Schaffner (2008), van Doorslaer (2009), on the challenges in journalistic translation research, namely the difficulty in recognizing the 
reconstruction process and language transfer in the news writing process. In a number of studies on journalistic translation, it is often mentioned about the invisibility of translation, that is, where translation is not considered part of the news writing process. Even people who carry out translation activities do not call themselves translators, but journalists. However this is not true in all cases. There are many news writers who are aware of the practice of translation as the main activity in news writing.

Another fact found in news translation is the difficulty of tracing the source text. One translated news is translated from more than one source text, although, again, this is not always the case. As a result, the relationship between the translated text and the source text is often questioned. Guerrero in [1] also questions this by pointing out the fact that news texts often do not include the author. Valdeon [3] mentions that there are stable and unstable sources in news translation. Editorials and opinions are examples of stable sources, while news genres, such as interviews, are examples of unstable sources.

Another criticism often directed to media translation is the big influence of extra-linguistic factors on translation activities placing editing as an inseparable part of translation activities. The magnitude of the changes made to the source text due to editing made a number of translation scholars propose alternative terms to subtitute the term "translation" [1]. Stetting (1989) proposed the term transediting to show the character of news translation. The term transediting is followed by a number of researchers to replace the term translation done in the world of journalism. Hursti (2001) stated that in news translation, editing and translation are not only always present but also have an equally important and highly interrelated position. Valdeon (2005) conducted a study using a textual approach which concluded that changes made in translation are a translation strategy. Meanwhile in other research (2008), which not only uses a textual approach but also contextual, apart from the existence of a translation strategy, the changes that occur are also due to ideological implications in news translation. Bani (2006) distinguishes changes made in news translation into two, namely textual manipulation by the editorial team and translation strategies. Kang (2007) states that the changes that occur in news translation are the result of collaboration between people with different roles involved in language transfer, cultural adaptation, proofreading, revision, naturalization, editing, and other textual processes that are carried out repeatedly and cyclically. Aktan and Nohl (2010) use the term trans-editing to describe the translation and editing process carried out by journalists / translators. They concluded 5 typical patterns of trans-editing, namely: adding information (mainly to explain), modifying semantic meaning (stylistic adaptation and semantic shifting), reducing information (eliminating idioms), increasing understanding by eliminating irrelevant parts, and input from the editor.

\section{News Translation as A Translation}

The various specificities and challenges described in the previous section cannot move news translation from its position as part of translation. It is true that news translation has special characteristics that affect the translation practices, but presumably it is shared by other types of translation. Therefore, it should not be used to question the existence of news translation as a form of translation.

Schaffner [4] highlights this in his writing entitled "Rethinking Transediting". Schaffner disagrees with the use of the term "transediting" to replace the term "translation". If the term transediting is used in place of the term translation, it is likely that translation will continue to 
be understood in a narrow sense as a word-for-word transfer process. Translation of journalistic texts have a very complex and varied process, and replacing the word "translation" with other terms is not a solution. Schaffner said that the use of new terms such as transediting, transadaptation, and transcreation serves to increase awareness of the complexity of the translation process that makes people rethink their traditional views on translation.

Various changes made to the source text, at the micro and macro levels are an integral part of any type of translation. These changes occur due to differences in the linguistic system of the source language and target language, and more often it is a result of consideration of the target reader. Apart from emphasizing message transfer, as with other types of translation, news translation which is a type of communicative translation, also places great emphasis on the problem of the effect that a translation has on the reader. Readers of translated news do not expect ambiguity in the translated text, and expect the transfer of elements of the source language into their culture and language [5]. Therefore, various changes are often made by translators to ensure that translation can carry out its function as a communication tool. In general, the changes can be classified into two main cases. First, changes made as an attempt by the translator to overcome various translation problems and achieve a functional translation, which is called translation strategy. Second, changes made as adjustments made by editors related to journalistic principles of media institutions.

News translation research should pay close attention to specific facts typical of this type of translation to be able to show a clear position of the study being carried out, so that it can make a real contribution to the study of translation. In order to truly understand both the process and product of media translation, Schaffner [4] suggests considering several things, namely the overall framework of the activities carried out by the translator as well as the policies and ideology that underlie these activities.

\section{Parallelism of Source Text with Target Text in News Translation}

Ensuring good access to source texts is an important first thing to do when conducting a news translation study because as discussed earlier, this is a matter that is often questioned. In addition, the existence of the source text which is parallel to the translated text becomes the main marker of a translated work. Thus, it is only natural that the parallelism between the source text and the translated text must be ensured.

Davier \& van Doorslaer [2] categorizes the parallelism of source text and target text into two types, namely corpora with identified source text and target text relationships, and corpora with unidentified source text and target text relationships. The first type of corpora can be found in media that use one to one translation, which means that one translated text is taken from one source text. In this one to one translation, the source text and target text can be analyzed in a classic and valid way.

One to one translation is rarely found in the translation of headlines. Valdeon [3] gave an example that one-to-one translation is mostly done on the translation of editorial or opinion pages. In Valdeon's term, editorial translation or opinion is included in a stable translation source.

One to one translation is also rare in the context of the media in Indonesia, especially in the translation of headlines. This can be observed on newspaper pages broadcasting international news. On a page that is specially provided for news from abroad, there are usually one or two headlines and several short news stories. In headlines that have a bigger space, at the end of the 
news, the identity of the news source appears, which usually consists of two or more sources. The use of more than one source text, according to several studies, is to add news details that are not found in one source. News sources are usually news wires subscribed to by the media institution. A media institution typically subscribes to more than one news wire. One to one translation in editorial translation is also very rare in the media in Indonesia.

Meanwhile, corpora with the unidentified relationship between source text and target text can be studied with the source text that is being held (texts that are considered source texts). Such corpora are called multilingual corpora. The source text is then matched with the translated text with criteria such as genre, time of writing, etc. (Johansson in [2]).

From the various opinions about parallelism that have been discussed, there are some important notes that must be considered from the translation of news in the media in Indonesia. Although most news translations in the media in Indonesia do not use the one-to-one translation type, the corpora type used is also not the corpora type that does not have a source text, or whose source text should be generated. In several studies that have been conducted, it is evident that several source texts are used by the translators to be rearranged in the form of translated texts.

From these facts, it appears that news translation can be studied well because the source text can be traced by researchers. Tracking is carried out by the researcher using data from the informant, namely the translator himself. From it, data on source texts can be obtained, which can then be compared with the translated news version in order to reveal various things that are usually the focus of translation studies, such as changes in translation, translation techniques, and translation quality.

\section{A Holistic Approach in Researches on News Translation}

Given the complexity and variety of activities involved in news translation, a combination of approaches is needed to accommodate the linguistic and extra-linguistic aspects in it. Arguing about the appropriate terms to represent the complexity of news translation will be futile if it is not followed by further investigation of the role of translation in the production of journalistic texts [2].

The nature of news translation must be understood textually and contextually. Textually, news is part of a narrative genre that has a specific function, a certain text structure, and certain linguistic characteristics. These facts are important things to pay attention to when comparing the translated text with the source text. Contextually, news translation is a series of processes that involve many actors, each of which influences the various activities that occur. In news translation, there are two main actors, namely the translator, and the editorial board acting as the editor. Changes that occur in the translation process should not be immediately questioned to the translators because they could be made by the editor.

The nature of news translation determines the translation method used, which includes the way in which translation is done at a macro level. This nature also brings about various translation problems which will be resolved by translators using translation techniques. Although translation techniques are used in general to solve problems that arise in any type of translation, not only news translation, knowledge of the nature of news translation can help researchers to more fairly assess the translation techniques used by translators.

The large social dimension in news translation led to the emergence of translation research using social science approaches in the 1990s known as the cultural turn (a shift in the paradigm of translation research from a traditional approach centered on source texts, to an approach that 
was highly inspired by cultural studies, sociology, anthropology, and others [6]. However, in the context of translation studies in Indonesia which is part of linguistics, the use of pure social or cultural science without using linguistics is not yet acceptable. In this case, the combination of linguistic studies with studies from other related fields becomes an intermediate solution to accommodate the large social dimension in news translation.

\section{Conclusion}

The dynamics of news translation in the form of criticism and suggestions that arise from a number of studies is more appropriate if interpreted as a challenge. If it can be resolved properly, these challenges will contribute to the existence and usefulness of news translation. It is translation scholars and researchers who can conquer this challenge by correcting the weaknesses shown in the criticisms given, and by pursuing higher quality studies by paying attention to the various aspects that are typical of news translation. This will increase the theoretical and practical contribution of news translation.

\section{References}

[1] Valdeon, Roberto A. Fifteen years of journalistic translation research and more. 2015. Perspectives: Studies in Translatology, 23 (4), pp. 634-662.

[2] Davier, Lucile \& Doorslaer, Luc Van. 2018. Translation without A Source Text: Methodological Issues in News Translation. 2015. Across Languages and Cultures 19 (2), pp. 241-257.

[3] Valdeon, Roberto A. (Un)stable Sources, Translation and News Production. 2015. Target 27:3, pp. 440-453

[4] Schaffner, Christina. Rethinking Transediting. 2012. Meta, 57 (4), pp. 866-883

[5] Nababan, M.R. Teori Menerjemah Bahasa Inggris. Yogyakarta: Pustaka Pelajar; 2003. 175 pages.

[6] Schaffner, Cristina \& Bassnett, Susan. Political Discourse, Media and Translation. Newcastle: Cambridge Scholars Publishing; 2010. 260 pages 